\title{
Incidência de lesão por pressão e avaliação do risco pela escala ELPO: estudo observacional
}

\author{
Incidence of pressure ulcer and risk assessment using the ELPO scale: observational study \\ Incidencia de úlcera por presión y evaluación de riesgos mediante la escala ELPO: estudio
}

observacional

Recebido: 02/11/2021 | Revisado: 15/11/2021 | Aceito: 18/11/2021 | Publicado: 28/11/2021

\author{
Daniella Cristina Julio Lima \\ ORCID: https://orcid.org/0000-0002-5950-3564 \\ Universidade Federal do Rio de Janeiro, Brasil \\ E-mail: daniellacristina.julio@yahoo.com.br \\ Karina Chamma Di Piero \\ ORCID: https://orcid.org/0000-0002-0102-6785 \\ Universidade Federal do Rio de Janeiro, Brasil \\ E-mail: kadipiero@gmail.com \\ Cecília Maria Izidoro Pinto \\ ORCID: https://orcid.org/0000-0003-2433-2811 \\ Universidade Federal do Rio de Janeiro, Brasil \\ E-mail: cecilia.izidoro@gmail.com \\ Camila Mendonça de Moraes \\ ORCID: https://orcid.org/0000-0001-5544-8409 \\ Universidade Federal do Rio de Janeiro, Brasil \\ E-mail: camila@macae.ufrj.br
}

\begin{abstract}
Resumo
Objetivo: Avaliar o risco para desenvolvimento de lesões por pressão relacionada ao posicionamento cirúrgico e sua incidência no centro cirúrgico de um Hospital Universitário do Rio de Janeiro, por meio da escala de avaliação de riscos para o desenvolvimento de lesões decorrentes do posicionamento cirúrgico do paciente (ELPO). Metodologia: Estudo observacional, tipo coorte, com análise quantitativa. Dados coletados entre setembro e novembro de 2018, em três etapas: visita de enfermagem pré-operatória, avaliação de risco intraoperatório e acompanhamento pós-operatório. Resultados: Dos 52 pacientes incluídos no estudo, 18 (34,6\%) foram classificados de alto risco pela ELPO e 34 $(65,4 \%)$ de baixo risco. Dos pacientes avaliados, quatro (8\%) evoluíram com uma lesão por pressão cada no período pós-operatório imediato após cirurgias com mais de $4 \mathrm{~h}$, em uso de anestesia geral e em sua maioria em posição supina. A incidência destas lesões foi 7,69\%. Conclusão: A escala foi útil para avaliação de risco e permitiu otimizar ações preventivas para lesões por pressão. Recomenda-se e corrobora-se a necessidade do uso da escala ELPO na prática clínica visando prever o risco de desenvolvimento de lesões por pressão no paciente cirúrgico e consequentemente a implantação de medidas preventivas relacionadas a cada situação cirúrgica.
\end{abstract}

Palavras-chave: Lesão por pressão; Segurança do paciente; Centros cirúrgicos; Posicionamento do paciente; Incidência.

\begin{abstract}
Objective: To evaluate the risk for developing pressure ulcers and its incidence in the surgical center of a University Hospital in Rio de Janeiro by using the risk assessment scale for the development of injuries resulting from surgical positioning of the patient (ELPO). Methodology: Cohort observational descriptive exploratory study with quantitative analysis. Data collected between september and november 2018 in three steps: preoperative nursing visit, evaluation of the perioperative moment and postoperative follow-up. Results: Fifty two patients included in the study, 18 $(34,6 \%)$ were classified as high risk by the ELPO and $34(65,4 \%)$ as low risk. Of the patients evaluated, four $(8 \%)$ evolved with one pressure ulcer each in the immediate postoperative period after surgeries that lasted more than 4 hours, under general anesthesia and mostly in the supine position. The incidence of these injuries was $7.69 \%$. Conclusion: The scale was useful for risk assessment and allowed to optimize preventive actions for pressure ulcer. It is recommended the need to use the ELPO scale in clinical practice in order to predict the risk of developing pressure ulcer in surgical patients and the implementation of preventive measures related to each surgical situation.
\end{abstract}

Keywords: Pressure ulcer; Patient safety; Surgicenters; Patient positioning; Incidence. 


\begin{abstract}
Resumen
Objetivo: Evaluar el riesgo de desarrollar úlceras por presión y su incidencia en el centro quirúrgico de un Hospital Universitario de Rio de Janeiro mediante el uso de la escala de evaluación de riesgo para el desarrollo de lesiones resultantes de la posición quirúrgica del paciente (ELPO). Metodología: Estudio observacional descriptivo exploratorio de cohorte con análisis cuantitativo. Datos recolectados entre septiembre y noviembre de 2018 en tres pasos: visita de enfermería preoperatoria, evaluación del momento perioperatorio y seguimiento postoperatorio. Resultados: Cincuenta y dos pacientes incluidos en el estudio, 18 (34,6\%) fueron clasificados como de alto riesgo por la ELPO y $34(65,4 \%)$ como de bajo riesgo. De los pacientes evaluados, cuatro $(8 \%)$ evolucionaron con una úlcera por presión cada uno en el postoperatorio inmediato después de cirugías que duraron más de 4 horas, em uso de anestesia general y mayoritariamente en decúbito supino. La incidencia de estas lesiones fue del 7,69\%. Conclusión: La escala fue útil para la evaluación de riesgos y permitió optimizar las acciones preventivas para úlcera por presión. Se recomienda la necesidad de utilizar la escala ELPO en la práctica clínica para predecir el riesgo de desarrollar úlcera por presión en pacientes quirúrgicos y la implementación de medidas preventivas relacionadas con cada situación quirúrgica.
\end{abstract}

Palabras clave: Úlcera por presión; Seguridad del paciente; Centros quirúrgicos; Posicionamiento del paciente; Incidencia.

\title{
1. Introdução
}

O enfermeiro perioperatório é responsável pelo planejamento e implementação de ações de enfermagem que minimizem ou possibilitem a prevenção de complicações decorrentes do procedimento anestésico-cirúrgico, visando medidas de segurança e conforto considerando a individualidade de cada paciente (SOBECC, 2017). Neste contexto, este profissional atua como integrante essencial da equipe multiprofissional para o desenvolvimento de um plano de cuidados que considere o tipo de anestesia e cirurgia, o tempo cirúrgico, a técnica cirúrgica utilizada, o posicionamento durante a cirurgia, a idade e as condições gerais de saúde do paciente (Nascimento \& Rodrigues, 2020; SOBECC, 2017).

Dentre as ações que a equipe desenvolve no período intraoperatório, destaca-se o posicionamento cirúrgico, procedimento importante para o bom desenvolvimento do ato operatório, o qual tem o objetivo de promover ótima exposição do sítio a ser operado (Trevilato et al., 2018). Com o avanço das práticas cirúrgicas e considerando que a cirurgia pode ser realizada em qualquer região do corpo, existem variedades de configurações de posicionamento que implicam em alterações do alinhamento corporal e consequentemente podem ocasionar lesões e complicações no paciente anestesiado (Oliveira et al., 2017).

O risco para lesão relacionada ao posicionamento cirúrgico é um diagnóstico de enfermagem frequente e que pode ser observado em 100\% dos pacientes dependendo do tipo de cirurgia realizada (Trevilato et al., 2018). As complicações decorrentes do posicionamento podem acontecer nos sistemas circulatório, respiratório, osteoarticular, muscular, neurológico e tegumentar (Buso et al., 2021). No presente estudo destacaremos as lesões tegumentares, por serem eventos adversos decorrentes do posicionamento que normalmente aparecem de 24 a 72 horas após o procedimento cirúrgico e dependem das intervenções da equipe intraoperatória para impedir seu desenvolvimento (Bezerra et al., 2020; Lopes et al., 2016).

A ocorrência de lesões tegumentares, em especial as lesões por pressão (LP), possuem impacto significativo para os pacientes, suas famílias e o sistema de saúde. Sabe-se que este tipo de evento repercute em custos para o governo, além de afetar fisicamente, emocionalmente e socialmente o indivíduo acometido e sua família, bem como na qualidade de vida destes (Jesus et al., 2020).

No que tange a qualidade em saúde e segurança do paciente, os eventos adversos têm sido considerados indicadores de qualidade de saúde, visto que tem associação com a assistência prestada, bem como, implica em altos custos para o sistema de saúde (Jesus et al., 2020). Segundo a definição de segurança do paciente da Organização Mundial de Saúde adotada pela Portaria no 529 (2013) e pelo Programa Nacional de Segurança do Paciente do Ministério da Saúde (PNSP, 2014), segurança do paciente significa "reduzir a um mínimo aceitável o risco de dano desnecessário associado ao cuidado de saúde". 
A qualidade em saúde e a segurança do paciente são aspectos fundamentais para garantir uma assistência mais efetiva, eficiente, igualitária e livre de danos. A melhoria destes dois pontos, consequentemente, diminui a incidência de eventos adversos tendo em vista o conhecimento sobre a importância de melhorar a qualidade dos cuidados prestados e a necessidade de colocá-los em prática (PNSP, 2014).

No contexto cirúrgico, é necessário amenizar as possíveis complicações oriundas dos procedimentos anestésicocirúrgicos (Trevilato et al., 2018). Logo é importante que o posicionamento do paciente cirúrgico seja realizado de forma segura e confortável a fim de prevenir possíveis complicações advindas do posicionamento, bem como os demais cuidados pertinentes ao paciente cirúrgico (Bezerra et al., 2020; Lopes et al., 2016).

Em caráter complementar à segurança do paciente, a utilização de escalas de medição de risco auxilia os profissionais de saúde na identificação de fatores predisponentes para o desenvolvimento de lesões e incorporação de medidas preventivas (Caetano, 2018; Eskildesen et al., 2019; Lopes et al., 2016).

Sendo assim, destacamos a escala ELPO (Escala de Avaliação de Risco para o Desenvolvimento de Lesões Decorrentes do Posicionamento Cirúrgico) validada em 2013 que permite predizer o risco de lesões decorrentes do procedimento cirúrgico. Assim, determinando um perfil da clientela assistida e permitindo a equipe de saúde planejar a assistência de forma individualizada, sistematizada e segura (Lopes et al., 2016).

Esta escala foi planejada com base em evidências científicas que indicam as implicações fisiológicas e as possíveis complicações no pós-operatório decorrente do posicionamento cirúrgico do paciente no período intraoperatório, sendo a escala mais adequada atualmente para a avaliação do risco nos pacientes cirúrgicos (Eskildesen et. al, 2019; Lopes et al., 2016; Miranda et al., 2016; Oliveira et al., 2019).

A ELPO é recomendada para ser aplicada durante o posicionamento do paciente na mesa cirúrgica e possui sete itens de avaliação: tipo de posição cirúrgica, tempo de cirurgia, tipo de anestesia, superfície de suporte, posição dos membros, comorbidades e idade do paciente. Cada item apresenta uma pontuação que varia de um a cinco pontos, sendo o escore total de 7 a 35 pontos. Quanto maior o escore em que o paciente é classificado, maior o risco de desenvolvimento de lesões decorrentes do posicionamento cirúrgico. A classificação em alto ou baixo risco permite estabelecer qual é o paciente que os profissionais de saúde deverão ter mais cautela e observação durante a execução do posicionamento, a fim de prevenir as complicações associadas ao procedimento (Lopes et al., 2016).

Sendo assim, o objetivo do estudo foi avaliar o risco de desenvolvimento de lesões por pressão relacionadas ao posicionamento cirúrgico e a incidência destas no centro cirúrgico de um hospital universitário do município do Rio de Janeiro em todas as especialidades cirúrgicas disponíveis neste estabelecimento a partir da escala ELPO.

\section{Metodologia}

Trata-se de um estudo de coorte, observacional, descritivo exploratório com análise quantitativa. Conforme Gil (2019), os estudos de coorte fazem parte de um tipo específico do estudo observacional, que investiga, observa e acompanha um grupo exposto e outro não exposto a um determinado fator de risco por um período de tempo, a fim de explorar os efeitos da exposição sobre o objeto de interesse, cujo cunho descritivo examina a incidência do evento, que varia de acordo com determinadas características. A análise quantitativa permite mensurar os dados objetivos da pesquisa.

O recrutamento dos pacientes e a posterior coleta de dados ocorreram entre setembro e novembro de 2018. A pesquisa foi aprovada pelo Comitê de Ética e Pesquisa (CEP) da Instituição (CAAE número 90268518.9.0000.5257) conforme a Resolução no 466 (2012) do Conselho Nacional de Saúde.

O local escolhido foi o centro cirúrgico de um hospital universitário do município do Rio de Janeiro devido a característica da instituição, que realiza cirurgias de pequeno, médio e grande porte, em diversas especialidades cirúrgicas 
como cirurgia geral, otorrinolaringologia, cirurgia plástica, neurocirurgia, ortopedia, cirurgia vascular, cardíaca, proctologia, urologia, ginecologia e cirurgia oral/buco maxilo.

Foram incluídos pacientes hospitalizados nos setores de internação com idade igual ou superior a 18 anos, submetidos à cirurgia eletiva, no hospital selecionado para o estudo.

Os critérios de exclusão foram pacientes com necessidade de cirurgia de emergência, uma vez que era necessário avaliação pré-operatória para inspeção da pele, leitura, e explicação da pesquisa e assinatura do Termo de Consentimento Livre e Esclarecido (TCLE) pelo próprio paciente ou seu responsável legal.

A seleção dos participantes ocorreu seguindo as etapas: verificação do mapa cirúrgico do dia para conhecimento dos pacientes e cirurgias propostas, onde foi considerada a especialidade cirúrgica do paciente para categorização da amostra e seleção dos pacientes conforme critérios de inclusão.

Em caso de paciente elegível para o estudo, fora realizado entrevista, avaliação do paciente e preenchimento da Ficha de Visita Pré-operatória conforme visita de enfermagem pré-operatória (rotina na instituição). Neste momento, também ocorreu a orientação sobre a pesquisa, leitura e assinatura do TCLE pelo próprio participante ou seu responsável legal, além de orientações de enfermagem pertinentes ao procedimento cirúrgico a ser realizado.

Durante a visita pré-operatória, ocorrida 24 horas antes da segunda etapa, foi realizada a inspeção da pele do paciente, registrando a integridade da pele e possíveis lesões prévias, bem como avaliação do risco de LP pela escala Braden (rotina na instituição). Tais dados foram registrados na Ficha de Visita Pré-operatória da instituição.

A segunda etapa consistiu no acompanhamento do paciente da sala operatória e no período intraoperatório até a sala de Recuperação Pós-Anestésica para avaliação do risco para desenvolvimento de LP, mediante a utilização e aplicação da escala ELPO. Nesta fase, foi observado o posicionamento do paciente pela equipe cirúrgica e calculado o risco de LP relacionada ao posicionamento cirúrgico do paciente, conforme a escala ELPO, sendo o tempo cirurgia estimado e recalculado ao final do procedimento, conforme recomendação da prática clínica da ELPO. Ainda nesta etapa ocorreu a categorização dos pacientes de acordo com os itens da ELPO (Lopes et al., 2016) em comorbidade, idade, especialidade cirúrgica, posição cirúrgica, tipo de anestesia, tempo cirúrgico, superfície de suporte, tempo de acompanhamento pós cirurgia e risco de desenvolvimento de LP, conforme Figura 1. 
Figura 1. Escala de Avaliação de Risco para o Desenvolvimento de Lesões Decorrentes do Posicionamento Cirúrgico. Rio de janeiro $(\mathrm{RJ})-2013$.

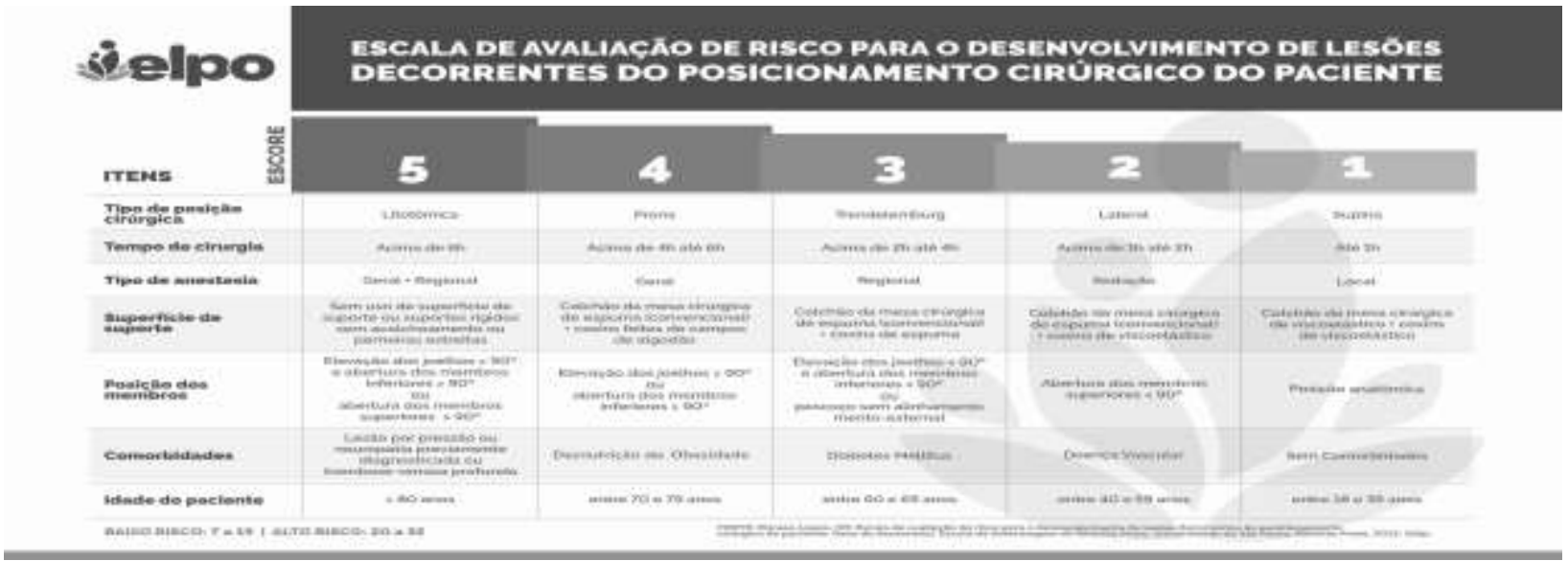

Fonte: Moraes-Lopes, CM. Escala de avaliação de risco para o desenvolvimento de lesões decorrentes do posicionamento cirúrgico do paciente. (tese de doutorado). Escola de Enfermagem de Ribeirão Preto, Universidade de São Paulo, Ribeirão Preto, 2013.156 p.

O tempo de acompanhamento no pós-operatório foi dividido em quatro períodos conforme a seguinte divisão estabelecida durante o período de internação hospitalar: menor que $12 \mathrm{~h}$, entre $12 \mathrm{~h}$ e $24 \mathrm{~h}$, a partir de $24 \mathrm{~h}$ até $48 \mathrm{~h}$ e a partir de $48 \mathrm{~h}$ até $72 \mathrm{~h}$.

A terceira e última etapa foi a visita de enfermagem no pós-operatório com o objetivo de verificar a ocorrência de lesões decorrentes do posicionamento cirúrgico. Foi realizado no período pós-operatório imediato e diariamente por até 72 horas após o procedimento cirúrgico, a fim de inspecionar toda a pele do paciente, em especial as áreas que ficaram em contato com a superfície de suporte e mesa operatória, de acordo com o tipo de posicionamento que este permaneceu no intraoperatório. Desta forma, os participantes foram seguidos desde o período pré-operatório, transoperatório e pós-operatório (até 72 horas) seguindo essas três etapas.

Nos casos de ocorrência de lesões, estas foram avaliadas quanto ao estágio conforme classificação do National Pressure Injury Advisory Panel (NPIAP, 2016) e encaminhado parecer técnico para avaliação e conduta especializada pela comissão de pele da instituição (rotina hospitalar), inclusive com acompanhamento ambulatorial após a alta hospitalar.

Cabe ressaltar que quaisquer alterações na condição clínica do paciente no período pós-operatório até $72 \mathrm{~h}$ após que pudesse interferir no risco de desenvolvimento de LP, foram avaliadas durante as visitas de enfermagem pelas pesquisadoras. Em caso de alteração clínica da pele, encaminhado novo parecer técnico para reavaliação da comissão de pele da instituição conforme rotina hospitalar.

Estabeleceu-se o período de até $72 \mathrm{~h}$ pós-procedimento cirúrgico para observação cutânea, pois as LP podem se manifestar até $72 \mathrm{~h}$ pós procedimento cirúrgico (Jesus et al., 2020). É importante salientar que todos os pacientes com risco de desenvolver LP no período pós-operatório tiveram como prescrição de enfermagem cuidados referentes a prevenção de LP como mudança de decúbito e descompressão, além de avaliação diária do risco pela escala de Braden nas unidades de internação cirúrgica (rotina hospitalar).

A amostra foi obtida por conveniência dentre os pacientes internados e que se submeteram a procedimentos cirúrgicos eletivos.

A incidência de LP foi calculada de acordo com a fórmula estatística descritiva da seguinte forma(Epidemiologia e serviços de saúde, 2009): 
Para a análise e tabulação dos dados de categorização da amostra foi utilizado o programa Microsoft Excel $^{\circledR}$. Os resultados foram distribuídos em gráficos, classificados e descritos de acordo com o percentual de risco alto ou baixo de ocorrência de LP decorrente do procedimento cirúrgico, incidência deste evento e perfil dos pacientes cirúrgicos, conforme as especialidades cirúrgicas analisadas em frequência relativa. Os dados categorizados foram calculados conforme estatística descritiva simples e descritos em valores absolutos e relativos.

Todos os pacientes cirúrgicos apresentam algum risco para desenvolvimento de LP. A depender de algumas variáveis como por exemplo, tempo de cirurgia, idade, tipo de anestesia e tipo de posicionamento cirúrgico, esse risco pode ser alto ou baixo, sendo os pacientes de alto risco mais susceptíveis a desenvolver LP.

\section{Resultados}

Incialmente, foram abordados 56 participantes potencialmente elegíveis. Foi realizada visita pré-operatória em todos esses pacientes. Entretanto, quatro não foram incluídos no estudo por motivo de piora clínica de um deles e cancelamento da cirurgia dos outros três. Assim, foram incluídos 52 pacientes no estudo que foram caracterizados na Tabela 1.

Tabela 1. Caracterização da amostra de pacientes avaliados quanto a dados demográficos e avaliação pré e perioperatória. Rio de Janeiro (RJ) - 2021.

\begin{tabular}{|c|c|c|c|}
\hline Variável & Amostra & Variável & Amostra \\
\hline Sexo & & Comorbidades & \\
\hline Feminino & $03(61,5 \%)$ & Sem comorbidades & $22(42,3 \%)$ \\
\hline Masculino & $20(38,5 \%)$ & Hipertensão Arterial Sistêmica & $24(46,2 \%)$ \\
\hline Idade & & Diabetes Mellitus & $05(9,6 \%)$ \\
\hline $18-39$ anos & $07(13,5 \%)$ & Obesidade & $01(1,9 \%)$ \\
\hline $40-59$ anos & $22(42,3 \%)$ & Especialidade Cirúrgica & \\
\hline $60-69$ anos & $19(36,5 \%)$ & Cirurgia Geral & $17(32,7 \%)$ \\
\hline $70-79$ anos & $01(1,9 \%)$ & Otorrinolaringologia & $02(3,8 \%)$ \\
\hline A partir de 80 anos & $03(5,8 \%)$ & Cirurgia Plástica & $05(9,6 \%)$ \\
\hline Posição Cirúrgica & & Neurocirurgia & $09(17,3 \%)$ \\
\hline Litotômica & $01(1,9 \%)$ & Ortopedia & $08(15,4 \%)$ \\
\hline Prona & $06(11,5 \%)$ & Cirurgia Vascular & $03(5,9 \%)$ \\
\hline Trendelemburg & $0 *$ & Cirurgia Cardíaca & $01(1,9 \%)$ \\
\hline Lateral & $02(3,9 \%)$ & Proctologia & $01(1,9 \%)$ \\
\hline Supina & $43(82,7 \%)$ & Urologia & $04(7,7 \%)$ \\
\hline Tipo de Anestesia & & Ginecologia & $01(1,9 \%)$ \\
\hline Local & $0 * *$ & Cirurgia Oral & $01(1,9 \%)$ \\
\hline Regional & $16(30,7 \%)$ & Tempo de Cirurgia & \\
\hline Geral & $34(65,4 \%)$ & Até 1 hora & $01(2,4 \%)$ \\
\hline Geral associada a & & Entre $1 \mathrm{~h}$ e $2 \mathrm{~h}$ & $05(9,6 \%)$ \\
\hline regional & $0 \angle(3,9 \%)$ & De $2 \mathrm{~h}$ até $4 \mathrm{~h}$ & $28(53,4 \%)$ \\
\hline Sedação & $0 * *$ & De $4 \mathrm{~h}$ até $6 \mathrm{~h}$ & $10(19,2 \%)$ \\
\hline & & Acima de $6 \mathrm{~h}$ & $08(15,4 \%)$ \\
\hline Acompanhamento no & & Risco de desenvolvimento de LP & \\
\hline pós-operatório & & & \\
\hline Até $12 \mathrm{~h}$ & $03(5,9 \%)$ & Alto Risco & $18(34,6 \%)$ \\
\hline De $12 \mathrm{~h}$ até $24 \mathrm{~h}$ & $19(36,5 \%)$ & & \\
\hline Maior que $24 \mathrm{~h}$ até $48 \mathrm{~h}$ & $12(23 \%)$ & Baixo Risco & $34(65,4 \%)$ \\
\hline De $48 \mathrm{~h}$ até $72 \mathrm{~h}$ & $18(34,6 \%)$ & & \\
\hline
\end{tabular}

\footnotetext{
* Nenhum paciente foi operado nesta posição. ** Nenhum paciente fez uso dos determinados tipos de anestesia. Fonte: Autores.
}

No que diz respeito a superfície de suporte, todos os 52 (100\%) pacientes foram operados em colchão convencional de espuma, uma vez que não há no setor outro tipo de colchão disponível. Em relação aos dispositivos e superfícies de suporte 
para alívio de pressão e descompressão, não há na instituição disponível dispositivos especializados no alívio da pressão para o intraoperatório, o que existe são adaptações com materiais de espuma, algodão e lençóis para a finalidade de reduzir a pressão de interface tecidual.

Do total da amostra, somente quatro pacientes desenvolveram LP decorrente do procedimento cirúrgico, sendo uma lesão em cada um destes pacientes. As características específicas estão dispostas na Tabela 2.

Tabela 2. Caracterização de pacientes que desenvolveram lesões por pressão. Rio de Janeiro (RJ) - 2021.

\begin{tabular}{cccc}
\hline Variável & Amostra & Variável & Amostra \\
\hline Idade & $01(25 \%)$ & Quantidade de lesões & $04(100 \%)$ \\
32 anos & $01(25 \%)$ & Quatro & $03(75 \%)$ \\
41 anos & $01(25 \%)$ & Especialidade Cirúrgica & $01(25 \%)$ \\
64 anos & $01(25 \%)$ & Cirurgia Geral & $01(25 \%)$ \\
82 anos & $04(100 \%)$ & Tempo Operatório & $03(75 \%)$ \\
Geral & De $4 \mathrm{~h}$ a $6 \mathrm{~h}$ & $02(50 \%)$ \\
Tipo de Anestesia & Acima de $6 \mathrm{~h}$ & $02(50 \%)$ \\
Classificação da LP & $01(50 \%)$ & Posição Cirúrgica & $01(25 \%)$ \\
Estágio 1 & Prona & $01(25 \%)$ \\
Estágio 2 & $01(25 \%)$ & Supina & $02(50 \%)$ \\
Tissular Profunda & $01(25 \%)$ & Comorbidades & Hipertensão arterial \\
Topografia da LP & $01(25 \%)$ & Obesidade & Sem comorbidades \\
Dorso & $01(25 \%)$ & $01(25 \%)$ & \multicolumn{2}{c}{ Mento } &
\end{tabular}

*Secundária a intubação orotraqueal. Fonte: Autores.

Quanto ao escore houve variação de 20 a 25 pontos nos pacientes classificados como alto risco e de 19 a 14 pontos nos pacientes classificados como baixo risco.

Desta forma, a incidência de lesões por pressão na amostra avaliada pela ELPO totalizou 7,69\%. Ainda, cabe ressaltar que não houve alteração no quadro clínico dos pacientes da amostra no período de pós-operatório imediato até 72h após ao procedimento cirúrgico que interferisse no risco de desenvolvimento de LP. Os pacientes que permaneceram como alto risco pela ELPO, foram observados diariamente por até $72 \mathrm{~h}$ com manutenção dos cuidados de enfermagem pertinentes a prevenção de LP como descompressão e mudança de decúbito (rotina institucional).

\section{Discussão}

Dentre os 52 pacientes, 18 (34, 6\%) apresentaram alto risco conforme a avaliação da escala ELPO, e os outros 34 (65, 4\%) pacientes apresentaram baixo risco. Posteriormente, 4 desses pacientes avaliados, aproximadamente $8 \%$, desenvolveram lesões. Das lesões por pressões desenvolvidas, 3 (75\%) foram pacientes em pacientes em pós-operatório de neurocirurgia.

Por conseguinte, o estadiamento de lesões mais encontrado foi de lesões por pressão estágio 1 que corrobora com evidências presentes na literatura que apontam que estas são as mais prevalentes no período pós-operatório (Caetano, 2018; Peixoto et al., 2019; Shafipour et al., 2016).

A apresentação das lesões pode estar relacionada à posição cirúrgica e ao tempo de cirurgia uma vez que quanto maior o escore na escala ELPO destes itens, associados à superfície de suporte, maior o risco à pele.

Quanto ao posicionamento cirúrgico o mais prevalente foi o decúbito dorsal. Estudos apontam como sendo a posição mais utilizada na maioria dos procedimentos cirúrgicos, além de ser a posição de escolha para anestesiar o paciente (Caetano, 2018; Oliveira et al., 2019; Trevilato et al., 2018). Um estudo realizado em um hospital privado da região sul do Brasil endossa os achando de que as posições cirúrgicas mais utilizadas foram supina ou dorsal e litotômica (Trevilato et al., 2018). 
Como limitações do estudo, o período curto de coleta de dados e as cirurgias suspensas de alguns pacientes limitaram a abrangência da amostra, consequentemente interferiram no perfil dos pacientes cirúrgicos traçados e na incidência de lesões por pressão decorrentes dos procedimentos anestésico-cirúrgicos da instituição.

Além disso, os dados encontrados evidenciam que cirurgias de grande porte com maior tempo de duração geralmente determinam mais dor e imobilidade no pós-operatório. Desta forma, esses pacientes necessitam de maior atenção e cuidados preventivos de LP visto alto risco de desenvolvimento de lesões nesses casos. Corroborando com essa afirmação, estudos demonstram que quanto maior o tempo cirúrgico e o tipo de cirurgia, maior o risco de lesões de posicionamento, sendo as LP com maior evidência de aparição, conforme, também apresentado nesta pesquisa (Nascimento \& Rodrigues, 2020; Sandes et al., 2019; Trevilato et al., 2018).

Os achados convergem a dados da literatura científica que aponta o aumento do risco de acordo com a modificação e as implicações anatomo-fisiológicas que cada posição cirúrgica provoca no organismo do paciente. Nesse sentido, quanto maior a modificação do alinhamento corporal, maior pré-disposição para o desenvolvimento de complicações, sendo estas mais comuns no sistema circulatório, respiratório, neurológico e/ ou tegumentar (Jost et al., 2018; Lopes et al., 2016; Miranda et. al, 2016).

Uma peculiaridade apresentada, é que a maioria dos pacientes que apresentaram lesão, foram identificadas no pósoperatório imediato. Tal fato ocorre devido a imobilidade e falta de descompressão para alívio de pressão nas áreas de proeminências ósseas ou mesmo da ausência da utilização de uma superfície de suporte que conseguisse redistribuir melhor a pressão de interface tecidual, associadas ao tempo cirúrgico e porte da cirurgia que quanto maior e mais complexa, maiores os riscos de lesões de pele (Sandes et al., 2019). A anestesia também é um fator de risco implicado no surgimento de lesões por pressão, sendo a anestesia geral a mais associada a lesões tegumentares devido seu maior poder de sedação em comparação a outros fármacos anestésicos (Engels et al., 2016).

Tendo em vista a necessidade de prevenir estes agravos, torna-se imprescindível durante os cuidados de enfermagem, como parte da sistematização da assistência de enfermagem perioperatória, a avaliação de riscos, de forma individualizada, considerando as particularidades do procedimento e as necessidades do paciente, para a implementação de medidas preventivas conforme a cirurgia realizada, tempo cirúrgico e perfil de risco do paciente avaliado. Logo, a ELPO se torna uma ferramenta fundamental para uma assistência efetiva e baseada em evidências (Bezerra et al., 2020; Jost et al., 2018).

Algumas comorbidades implicam fragilidade dos sistemas do corpo do paciente, como doenças vasculares, respiratórias, neuropatias ou até desnutrição. Quanto mais graves forem as comorbidades que acometem o paciente, especialmente as doenças que acometem a perfusão e oxigenação tecidual, maior será o risco de desenvolvimento de lesões decorrentes do posicionamento cirúrgico do paciente (Buso et al., 2021).

A ELPO foi formulada com base nas implicações fisiológicas e as possíveis complicações no pós-operatório decorrente do posicionamento cirúrgico do paciente no período intraoperatório, sendo mais específica e sensível para esse período, permitindo a equipe de saúde prever ações preventivas de lesões e eventos adversos preveníveis (Lopes et al., 2016; Miranda et al., 2016).

Verificou-se a inexistência de superfícies de suporte redistribuidores de pressão na instituição proponente, fato que pode ter contribuído para o aumento da pressão de interface tecidual naqueles pacientes apontados em alto risco. Estudos demonstram que os dispositivos de suporte e aliviadores de pressão, apresentam diversos modelos e tamanhos disponíveis no mercado nacional, bem como diversos materiais componentes, como espuma, fluido viscoso, etc (Bezerra et al., 2020; Oliveira, 2017).

Tais dispositivos devem ser utilizados para evitar pontos de maior pressão e contato corporal, a fim de redistribuir a pressão e prevenir lesões por pressão. Para o intraoperatório são indicados os dispositivos que não atrapalham a estabilidade do 
campo a ser operado, sendo dessa forma estáticos. Dos dispositivos estáticos disponíveis e melhor indicados para o intraoperatório atualmente estão as superfícies compostas por gel de viscoelástico (Oliveira et al., 2018).

Adicionalmente, superfícies de suporte como perneiras e braçadeiras, devem ser largas e compostas por material que reduzem ou redistribuem a pressão para evitar pontos de pressão (Lopes et al., 2016).

Reforça-se diante disso, que para a prevenção, o ideal é a utilização de colchões que aliviam e equilibram a pressão, e que não proporcionem deformação do material, além do uso de coxins/posicionadores para a realização das manobras de descompressão pela equipe de saúde no intraoperatório (Oliveira et al., 2017; Oliveira, 2017). Ademais, deve-se realizar adequação da posição necessária para a realização da cirurgia a ser realizada de acordo com as especificidades e necessidades do paciente, mantendo sempre o alinhamento corporal, a proteção dos olhos (fechados), orelha sem dobras e nariz sem pontos de pressão, entre outros cuidados específicos de cada posição (Oliveira et al., 2019).

Pode-se perceber com a análise dessa amostra de pacientes que há necessidade de investir na cultura de segurança do paciente visto que ainda há falhas em sua continuidade. Além disto, há necessidade de investir em medidas de prevenção de riscos, pois mesmo considerando a implantação de um programa de segurança do paciente e cirurgia segura, ainda é escasso o uso de escalas preditivas de risco que tem vital relevância para estratificação do perfil de risco da clientela.

\section{Conclusão}

As lesões por pressão são eventos adversos considerados um problema de saúde pública, tendo em vista sua prevalência nas unidades de saúde e as consequências negativas que trazem para o paciente. Dentre elas, o aumento do tempo de internação, desconforto, dor, risco de infeção da ferida, consequentemente, necessidade de antibioticoterapia, podendo levar inclusive ao desfecho óbito. Tudo isso está associado ao aumento dos custos hospitalares.

A avaliação de risco para lesões decorrentes de procedimento cirúrgico em pacientes adultos pela escala ELPO mostrou-se confiável e útil para a prática clínica da enfermagem no contexto cirúrgico. Por meio deste instrumento, foi possível traçar um perfil de pacientes com maior risco de desenvolvimento de LP, além evidenciar que taxa de incidência de LP na amostra selecionada foi considerável.

Os achados evidenciam que com auxílio da escala é possível compreender melhor o nível de risco de cada paciente e assim, possibilitando traçar medidas preventivas mais otimizadas para este cuidado. Diante disto, reforça-se a importância de traçar estratégias preventivas visando manutenção da integridade da pele e evitar o desenvolvimento deste evento adverso decorrente do procedimento cirúrgico. O uso do instrumento preditivo de risco de LP mostrou grande contribuição para auxiliar essa assistência prestada pelos profissionais de saúde visto que norteia ações preventivas.

Embora ainda pouco utilizada na instituição do estudo, os resultados contribuem para que a utilização desta ferramenta seja implementada como rotina institucional, tendo em vista as vantagens de se proporcionar maior segurança aos pacientes, por meio de uma sistematização da assistência de enfermagem pautada em evidências. Ademais, se faz necessário a manutenção das ações preventivas em todas as etapas da hospitalização visando cultura de segurança do paciente.

Espera-se que este estudo contribua com novos dados e informações a respeito da ocorrência de LP decorrente dos procedimentos anestésico-cirúrgicos, bem como no âmbito da segurança do paciente, subsidiando futuras pesquisas com maior robustez e norteando profissionais da área de saúde a enfrentar este tipo de agravo, através de medidas preventivas de lesões por pressões nas unidades de centro cirúrgico, estimulando o uso de escalas de medição de risco que proporcionam ao profissional de saúde prever o risco e antecipar ações para evitar este tipo de agravo, tendo em vista a vulnerabilidade dos pacientes cirúrgicos. 
Contudo, sugere-se novos estudos a respeito deste tema, visando o fortalecimento das evidências científicas sobre a importância e o impacto positivo que as ferramentas utilizadas para prevenção de lesões e agravos como a escala ELPO, por exemplo, contribuem para a assistência em prol da segurança do paciente.

\section{Referências}

Associação Brasileira de Enfermeiros de Centro Cirúrgico, Recuperação Anestésica e Centro de Material e Esterilização (SOBECC). (2017). Diretrizes de práticas em enfermagem cirúrgica e processamento de produtos para a saúde. (7a ed.), SOBECC; Manole.

Bezerra, S. M. G., Brito, J. F. P., Lira, J. A. C., Barbosa, N. S., Carvalho, K. G. \& Sousa, L. S. (2020). Estratégias de enfermagem para prevenção de lesão por pressão em pacientes cirúrgicos. ESTIMA Braziliam Journal of Enterostomal Therapy, 18: e1020. https://doi.org/10.30886/estima.v18.793_PT

Buso, F. D. S., Ferreira, M. B. G., Felix, M. M. S., Galvão, C. M., Barichello, E. \& Barbosa, M. H. (2021). Lesão por pressão decorrente do posicionamento cirúrgico e fatores associados. Acta Paulista de Enfermagem, 34: eape0064. https://doi.org/10.37689/acta-ape/2021ao00642

Caetano, E. P. S. (2018). Risco para Lesões do Posicionamento Cirúrgico decorrentes da posição supina. (Dissertação de mestrado). Universidade de Minas Gerais, http://hdl.handle.net/1843/ENFC-B9AHDX

Engels, D., Austin, M. \& Mcnichol, L., \& Fencl, J. (2016). Pressure ulcers: factors contributing to their development in the OR. AORN Journal, v. 103(3): 271-81. https://aornjournal.onlinelibrary. wiley.com/doi/abs/10.1016/j.aorn.2016.01.008

Epidemiologia e serviços de saúde. (2009). Ministério da Saúde, 18 (2). Editora Atheneu. http: www.passeidireto.com/arquivo/45475884/epidemiologiamedronho

Eskildesen, L., Forti, A., Paião, L. \& Magri, M. A. (2019). Aplicação da escala ELPO em pacientes submetidos às cirurgias cardiovasculares. CuidArte Enfermagem, 13(2): 116-121. http:// www.webfipa.net/facfipa/ner/sumarios/cuidarte/2019v2/116.pdf

Gil, A. C. Como elaborar projetos de pesquisa. (2019). (6a ed.), Atlas.

Jesus, M. A. P., Pires, P. S., Biondo, C. S. \& Matos, R. M. (2020). Incidência de lesão por pressão em pacientes internados e fatores de risco associados. Revista Baiana de Enfermagem, 34. https://periodicos.ufba.br/index.php/enfermagem/article/view/36587

Jost, M. T., Viegas, K. \& Caregnato, R. C. A. (2018). Sistematização da assistência de enfermagem perioperatória: revisão integrativa. Revista SOBECC, 23(4): 218-225. https://doi.org/10.5327/Z1414-4425201800040009

Lopes, C. M. M., Haas, V.J., Dantas, R. A. S., Oliveira, C. G. \& Galvão, C. M. (2016). Escala de avaliação de risco para lesões decorrentes do posicionamento cirúrgico. Revista Latino Americana de Enfermagem, 24: e2704. http://www.scielo.br/pdf/rlae/v24/pt_0104-1169-rlae-24-02704.pdf

Portaria $n^{o} 529$ de 2013. (2013). Institui o Programa Nacional de Segurança do Paciente (PNSP). Diário Oficial da União da República Federativa do Brasil. Ministério da Saúde (Brasil).

Programa Nacional de Segurança do Paciente (PNSP). (2014). Ministério da Saúde; Fundação Oswaldo Cruz; Agência Nacional de Vigilância Sanitária. Brasília: Ministério da Saúde.

Miranda, A. B., Fogaça, A. R., Rizzetto, M. \& Lopes, L. C. C. (2016). Posicionamento cirúrgico: cuidados de enfermagem no transoperatório. Revista SOBECC, 21(1):52-58. http://files.bvs.br/upload/S/1414-4425/2016/v21n1/a5578.pdf

Nascimento, F. C. L. \& Rodrigues, M. C. S. (2020). Risco para lesões de posicionamento cirúrgico: validação da escala em hospital de reabilitação. Revista Latino Americana de Enfermagem, 26(2): 99-106. http://dx.doi.org/10.1590/1518-8345.2912.3261

National Pressure Injury Advisory Panel (NPIAP). (2016). NPIAP Pressure Injury Stages. http://cdn.ymaws.com/npiap.com/resource/re smgr/online_store/npiap_pressure_injury_stages.pdf

Oliveira, K. F. (2017). Superfícies de suporte para prevenção de lesões por pressão por posicionamento cirúrgico: um estudo experimental. (Tese de doutorado em Atenção à Saúde). Universidade Federal do Triângulo Mineiro, Uberaba.

Oliveira, K. F., Nascimento, K. G., Nicolussi, A. C., Chavaglia, S. R. R., Araújo, C. A. \& Barbosa, M. H. (2017). Support surfaces in the prevention of pressure ulcers in surgical patients: An integrative review. International Journal of Nursing Practice, 23: e12553. http//www.ncbi.nlm.gov/pubmed/28643855

Oliveira, K. F., Pires, P. S., De-Mattia, A. L., Barichello, E., Galvão, C. M., Araújo, C. A. \& Barbosa, M. H. (2018). Influence of support surfaces on the distribution of body interface pressure in surgical positioning. Revista Latino Americano de Enfermagem, 26:e3083. https://doi.org/10.1590/1518-8345.2692.3083

Oliveira, H. M. B. S., Santos, A. M. J. F., Madeira, M. Z. A., Andrade, E. M. L. R. \& Silva, G. R. F. (2019). Avaliação do risco para o desenvolvimento de lesões perioperatórias decorrentes do posicionamento cirúrgico. Revista Gaúcha de Enfermagem, 40: e20180114. https://doi.org/10.1590/19831447.2019.20180114

Peixoto, C. A., Ferreira, M. B. G., Felix, M. M. S., Pires P. S., Barichello, E. \& Barbosa, M. H. (2019). Classificação de risco de desenvolvimento de lesões decorrentes do posicionamento cirúrgico. Revista Latino Americana de Enfermagem, 27: e3117. http://0104-1169-rlae-27-e3117.pdf (scielo.br)

Resolução no 466 de 12 de dezembro de 2012. (2012). Diretrizes e normas regulamentadoras de pesquisas envolvendo seres humanos. Diário Oficial da União da República Federativa do Brasil. Ministério da Saúde (Brasil). Conselho Nacional de Saúde, 150 (112 Seção 1): 59-62. 
Research, Society and Development, v. 10, n. 15, e403101522704, 2021

(CC BY 4.0) | ISSN 2525-3409 | DOI: http://dx.doi.org/10.33448/rsd-v10i15.22704

Sandes, S. M. S., Costa, M. F., Santos, G. V., Freitas, L. P., Vasconcelos, A. C. P. \& Silva, L. S. L. (2019). Lesões provenientes de procedimento cirúrgico: fatores relacionados. Revista SOBECC, 24(3): 161-167. http://revista.sobecc.org.br/sobecc/article/view/479

Shafipour, V., Ramezanpour, E., Gorji, M. A. H. \& Moosazadeh, M. (2016). Prevalence of postoperative pressure ulcer: A systematic review and metaanalysis. Electronic Physician Journal, 8(11): 3170-3176. http://www.ephysician.ir/index.php/browse-issues/2016/11/507-3170

Trevilato, T. T., Melo, T. C., Fagundes, M. A. B. G. \& Caregnato, R. C. A. (2018). Posicionamento cirúrgico: prevalência de risco de lesões em pacientes cirúrgicos. Revista SOBECC, 23(3): 124-129. httpp://revista.sobecc.org.br/sobecc/article/view/398 